\title{
Velvety Plaques on the Abdomen and Extremities
}

\author{
Alana Deutsch, MD; Parth Patel, MD; Yevgeniy Balagula, MD
}
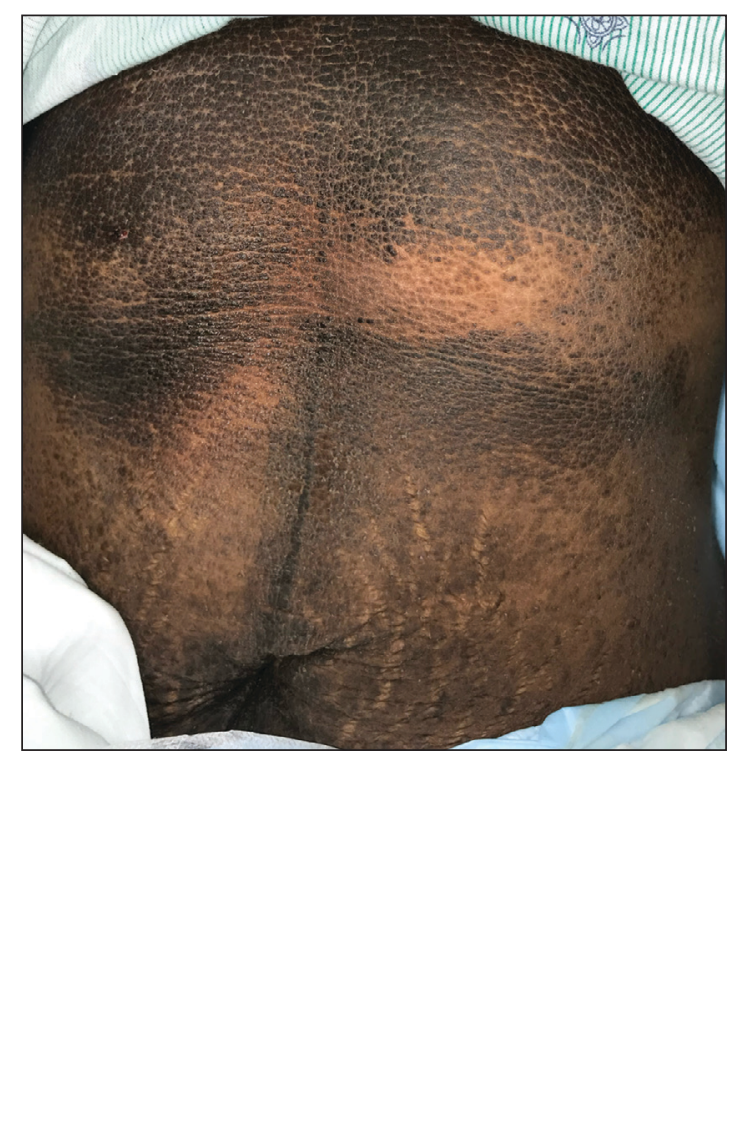

A 28-year-old woman was admitted to the medicine service with bilateral pedal numbness and ataxia, as well as an asymptomatic rash on the neck, chest, abdomen, and extremities of a few months' duration. The patient was seen by an outside dermatologist for the same rash 1 month prior, at which time a punch biopsy of the right forearm was suggestive of psoriasiform dermatitis; however, the rash failed to improve with topical ammonium lactate and corticosteroids. During the current admission, the patient was found to have low methylmalonic acid and vitamin $B_{1}$ levels; however, vitamin $B_{12}$, thyroid studies, rapid plasma reagin test, and inflammatory markers, as well as central and peripheral imaging and nerve conduction studies were normal.

Dermatology was consulted. Physical examination revealed retention hyperkeratosis on the neck that was wipeable with $70 \%$ isopropyl alcohol, as well as nonwipeable, thin, reticulated plaques on the mid chest and thick velvety plaques on the abdomen and bilateral extremities. There was notable sparing of areas with natural occlusion such as the back and body folds. A punch biopsy of the abdomen was performed.

\section{WHAT'S YOUR DIAGNOSIS?}
a. acanthosis nigricans
b. confluent and reticulated papillomatosis
c. dermatitis neglecta
d. psoriasis
e. terra firma-forme dermatosis 


\section{THE DIAGNOSIS: Dermatitis Neglecta}

A punch biopsy of the abdomen revealed hyperkeratosis and mild papillomatosis (Figure), which can be seen in dermatitis neglecta (DN) and acanthosis nigricans (AN) as well as confluent and reticulated papillomatosis (CARP). Due to the patient's history of mood and psychotic disorders, collateral information was obtained from the patient's family, who reported that the patient had a depressed mood in the last few months and was not showering or caring for herself during this period. There was no additional personal or family history of skin disease. Clinical and histopathologic findings led to a diagnosis of DN. Following recommendations for daily cleansing with soap and water along with topical ammonium lactate, near-complete resolution of the rash was achieved in 3 weeks.

Dermatitis neglecta, or unwashed dermatosis, is a skin condition that occurs secondary to poor hygiene, which was first reported in 1995 by Poskitt et al. ${ }^{1}$ Avoidance of washing in affected areas can be due to physical disability, pain after injury, neurological deficit, or psychologically induced fear or neglect. Sebum, sweat, corneocytes, and bacteria combine into compact adherent crusts of dirt, which appear as hyperkeratotic plaques with cornflakelike scale. ${ }^{2,3}$ Despite its innate simplicity, $\mathrm{DN}$ is a diagnostic challenge, as it clinically and histologically mimics other dermatoses including AN, terra firmaforme dermatosis, and CARP. ${ }^{2,4}$ Ultimately, the diagnosis of $\mathrm{DN}$ can be made when a history of poor hygiene is probable or elicited, and lesions can be removed with soap and water. Treatment of DN includes daily cleansing with soap and water; however, resistant lesions or extensive disease may require keratolytic agents, as in our patient. ${ }^{2-4}$ In contrast, terra firma-forme dermatosis, which may look similar, is not due to poor hygiene, and the lesions typically are resistant to soap and water, classically requiring isopropyl alcohol for removal. Overall, maintained awareness of $\mathrm{DN}$ is imperative, as early diagnosis can avoid unnecessary biopsies and more complex treatment measures as well as facilitate coordination of care when additional medical or psychiatric concerns are present.

Although the diagnoses of DN and terra firma-forme dermatosis can be distinguished based on the patient's clinical history and response to simple cleansing measures alone, the alternate diagnoses can be excluded based on different clinical distributions and response to other treatment modalities but sometimes may require clinicopathologic correlation for definitive diagnosis. Our patient had a biopsy diagnosis of psoriasiform dermatitis from an outside provider, but neither her clinical disease nor repeated histopathologic findings supported

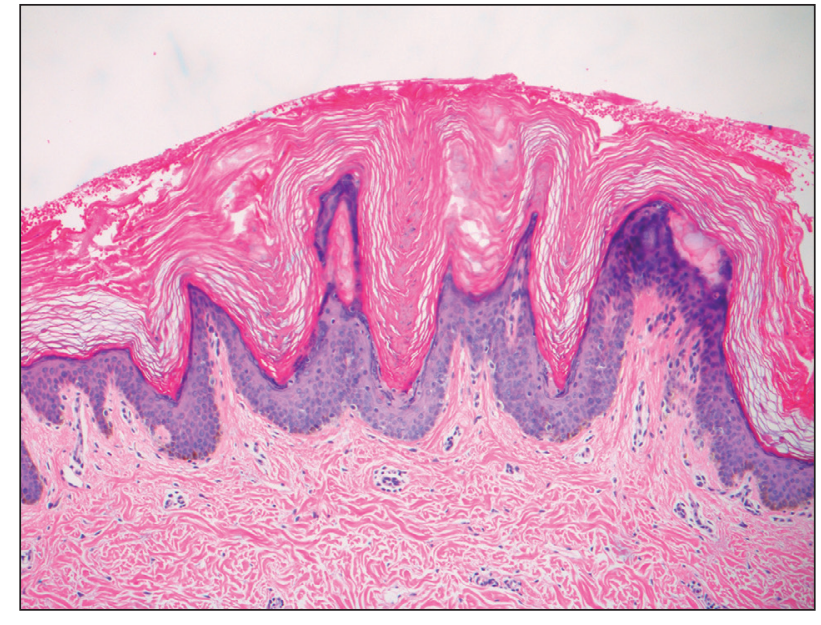

Dermatitis neglecta. Punch biopsy of the abdomen showed hyperkeratosis and mild papillomatosis (H\&E, original magnification $\times 10$ ).

a diagnosis of psoriasis or other classic psoriasiform dermatoses such as contact dermatitis, dermatophyte/ candidal infection, seborrheic dermatitis, pityriasis rubra pilaris, pityriasis rosea, scabies, or syphilis.

It is imperative to exclude alternative diagnoses because they can have systemic implications and can misguide treatment, as was done initially with our patient. Psoriasis vulgaris in its classic form is a chronic inflammatory skin disease that manifests as sharply demarcated, erythematous plaques with overlying thick silvery scale; it has the additional histologic findings of neutrophilic spongiform pustules in the epidermis, tortuous blood vessels in the papillary dermis, and neutrophils and parakeratosis in the stratum corneum. In its benign form, $\mathrm{AN}$ is associated with endocrinopathies, most commonly obesity and insulin-resistant diabetes mellitus, and presents as hyperkeratotic, velvety, hyperpigmented plaques typically limited to the neck and axillae. Malignant AN spontaneously arises in association with systemic malignancy and can be extensive and generalized. ${ }^{5}$ Treatment of AN primarily focuses on resolution of the underlying systemic disease; however, cosmetic treatment with topical or oral retinoids may hasten resolution of cutaneous disease. ${ }^{6}$ Confluent and reticulated papillomatosis is characterized by reticulated hyperkeratotic plaques with a common distribution over the central and upper trunk. Unlike DN and AN, which may occur at any age, CARP typically is seen in adolescents and young adults. ${ }^{7}$ There is no evidence-based gold standard for the management of CARP; however, the successful use of various antibiotics, antifungals, and retinoids-alone or in combination-has 
been reported. ${ }^{8}$ Overall, compared to the other entities in the differential diagnosis, DN easily can be prevented with consistent use of soap and water and may be underreported given the asymptomatic nature of the disease and the typical patient population.

\section{REFERENCES}

1. Poskitt L, Wayte J, Wojnarowska F, et al. 'Dermatitis neglecta': unwashed dermatosis. Br J Dermatol. 1995;132:827-829.

2. Perez-Rodriguez IM, Munoz-Garza FZ, Ocampo-Candiani J. An unusually severe case of dermatosis neglecta: a diagnostic challenge. Case Rep Dermatol. 2014;6:194-199.

3. Park JM, Roh MR, Kwon JE, et al. A case of generalized dermatitis neglecta mimicking psoriasis vulgaris. Arch Dermatol. 2010;146:1050-1051.
4. Lopes S, Vide J, Antunes I, et al. Dermatitis neglecta: a challenging diagnosis in psychodermatology. Acta Dermatovenerol Alp Pannonica Adriat. 2018;27:109-110.

5. Shah KR, Boland CR, Patel M, et al. Cutaneous manifestations of gastrointestinal disease: part I. J Am Acad Dermatol. 2013;68:189. e1-21; quiz 210.

6. Patel NU, Roach $\mathrm{C}$, Alinia $\mathrm{H}$, et al. Current treatment options for acanthosis nigricans. Clin Cosmet Investig Dermatol. 2018; 11:407-413.

7. Kurtyka DJ, Burke KT, DeKlotz CMC. Use of topical sirolimus (rapamycin) for treating confluent and reticulated papillomatosis. JAMA Dermatol. 2021;157:121-123.

8. Mufti A, Sachdeva M, Maliyar K, et al. Treatment outcomes in confluent and reticulated papillomatosis: a systematic review. J Am Acad Dermatol. 2021;84:825-829. 